\title{
A Rare Chondrosarcoma of the Larynx Presenting as Difficult Intubation: Report of a Case and Literature Review
}

\author{
Jaydev Sarma ${ }^{1, *}$, Srinivas M. Susarla ${ }^{2}$, William C. Faquin ${ }^{3}$ and Phillip C. Song ${ }^{4}$
}

\begin{abstract}
${ }^{I}$ Department of Anesthesia, Critical Care and Pain Medicine, Massachusetts General Hospital Anesthesia, Harvard Medical School, Boston, MA, USA; ${ }^{2}$ Department of Oral and Maxillofacial Surgery, Massachusetts General Hospital, Boston, MA, USA; ${ }^{3}$ Department of Pathology, Massachusetts General Hospital, Harvard Medical School, Boston, MA, USA; ${ }^{4}$ Staff Surgeon, Division of Laryngology, Department of Otolaryngology, Massachusetts Eye and Ear Infirmary, Harvard Medical School, Boston, MA, USA
\end{abstract}

\begin{abstract}
Chondrosarcomas of the larynx are rare tumors, representing less than $0.2 \%$ of all head and neck malignancies [1]. In this report, we present an unusual case of an undiagnosed chrondrosarcoma of the larynx presenting as difficult intubation in an otherwise asymptomatic patient. Difficult intubation in an otherwise asymptomatic patient has not been reported as an initial presentation of this tumor. Men are more commonly affected than women (3.6: 1), and the tumors typically present in the fifth or sixth decade of life. Patients may present with dyspnea, dysphagia, hoarseness of the voice, airway obstruction and some may have pain as a result of expansion of this tumor. The tumors almost always arise from hyaline cartilage, with the most common site of involvement the cricoid cartilage (75\%), specifically the posterior lamina, with the thyroid cartilage and arytenoid cartilage less frequently involved. Though locally invasive, these tumors are characterized by a low tendency for distant metastasis and the overall prognosis following excision is excellent [2].
\end{abstract}

Keywords: Difficult intubation, chondrosarcoma, larynx.

\section{CASE REPORT}

A 78 year old man was scheduled for a right knee replacement surgery. He had a past medical history of atrial fibrillation and hypertension and he was taking an angiotensin receptor blocker (Olmesartan), a thiazide diuretic (hydrochlorthiazide), and an anti-coagulant (Coumadin). He had no known drug allergies and had an unremarkable review of systems, including symptoms of dysphagia, odynophagia, stridor, dyspnea, or hoarseness. His medical history was likewise unremarkable with regard to smoking and alcohol intake. He was 71" tall and weighed $211 \mathrm{lbs}(\mathrm{BMI}=29.4$ $\mathrm{kg} / \mathrm{m}^{2}$ ), with a Mallampati class 111 airway. The thyromental distance was four finger breadths and he had good neck extension. He had a marked class III skeletal profile and the teeth were in good repair with no dentures or implants. His vital signs and laboratory workup were unremarkable. The patient refused spinal anesthesia. As such, the case was performed under general anesthesia.

The patient was premedicated with $2 \mathrm{mg}$ of Midazolam and $100 \mathrm{mcg}$ of Fentanyl. After adequate preoxygenation he was induced with $250 \mathrm{mg}$ of propofol and $100 \mathrm{mg}$ of succinylcholine. A first attempt at intubation was attempted by the nurse anesthetist using a Macintosh size 3 blade laryngoscope and was unsuccessful. During direct laryngoscopy on a the subsequent attempt by the staff anesthesiologist, using

*Address correspondence to this author at the Department of Anesthesia, Critical Care and Pain Medicine, Massachusetts General Hospital and Instructor in Anesthesia, Harvard Medical School, Boston, MA, USA; Tel: 6177247182; Fax: 6177264489; E-mail: jsarma@partners.org a Macintosh size 4 blade, the view was poor, but a small bulge under the right posterior vocal fold was visualized. The glottic aperture was deviated to the left and was narrowed (Fig. 1). A standard bougie was passed under direct vision through the glottic aperture and a $7.0 \mathrm{~mm}$ cuffed endotracheal tube was passed over the bougie into the trachea with intubation achieved after some difficulty. The rest of the case was uneventful. After the patient was fully reversed and was able to follow commands and was able to achieve good tidal volumes and airway pressures, the endotracheal tube was removed over a standard airway exchanger, allowing for the administration of oxygen. This was done in case the patient needed to be re-intubated emergently. A size 5 LMA was prepared for insertion as a back up. The extubation was completed uneventfully.

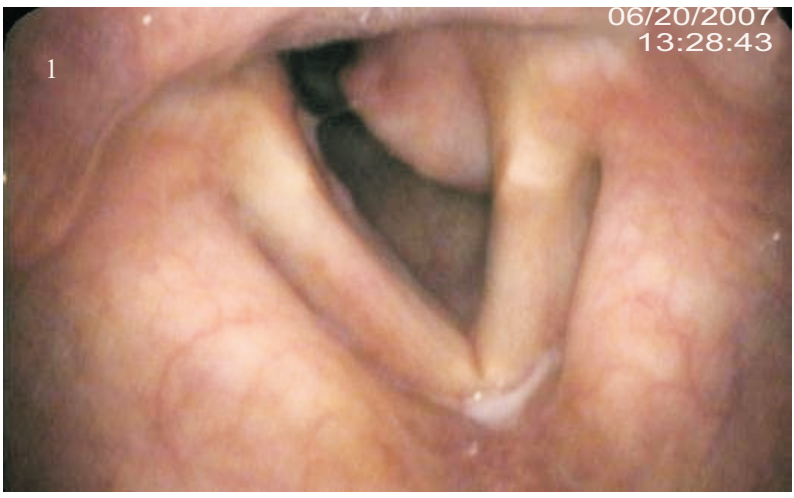

Fig. (1). Initial presentation of chondrosarcoma. Superior view from bronchoscope, demonstrating left sided subglottic soft tissue mass with associated narrowing of the glottic aperture. 
The patient was followed closely in the post operative recovery area for any signs of airway obstruction. He was given supplemental oxygen for an hour in the recovery area and was discharged to the general floor once his vital signs stabilized. The treating team spoke to the patient and his family members about the discovery of a probable laryngeal mass and a note describing the difficult intubation was given to patient. The orthopedic surgeon was informed about our findings and the patient was referred to a laryngologist (PCS) for further evaluation.

The patient subsequently had a CT scan (Fig. 2) and a MRI of the head and neck, which showed a mass at the level of the cricoid cartilage $3 \mathrm{~cm}$ in size in the right posterior paramedian region. He underwent a suspension microlaryngoscopic excision of the subglottic mass with carbon dioxide laser and a $3.5 \mathrm{~cm}$ tumor was removed. For this operation, the airway was secured using a $6.5 \mathrm{~mm}$ cuffed endotracheal with awake fiberoptic intubation. Anesthesia and recovery were uneventful.
Histologic examination of the laryngeal biopsy revealed fragments of mature hyaline-type cartilage as well as admixed areas of bone (Fig. 3). The hyaline cartilage was noted to be hypercellular with chondrocytes present within welldefined lacunae. Occasional chondrocyte nuclei demonstrated moderate enlargement with low-grade nuclear atypia (Fig. 4), with many areas of enchondral ossification are present. The overall histologic findings were diagnostic of a low-grade (grade 1/3), hyaline type chondrosarcoma of the larynx.

The patient subsequently underwent radiation therapy and his six months and one year follow ups at the laryngology clinic showed improved mobility of the right vocal fold with a mild disruption of the glottic closure and rotation toward the right (Fig. 5), but normal interior contour. There was moderate periarytenoid and post cricoid edema and the voice was mostly intact, with mild roughness. The patient had mild dysphagia following surgery and radiation therapy, which improved in the interval post-operative period to the

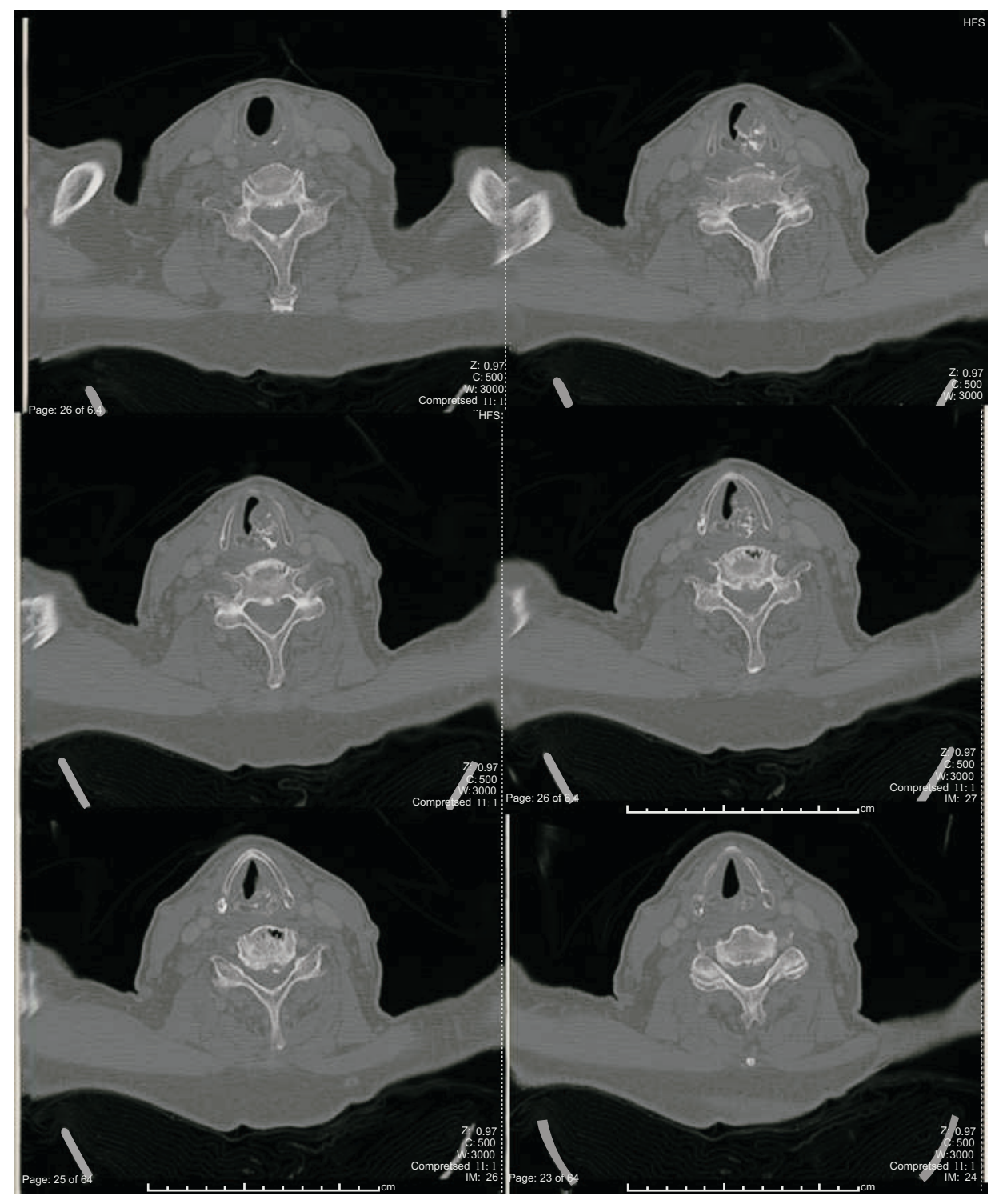

Fig. (2). Neck computed tomography, demonstrating laryngeal mass. The axial cuts above demonstrate the mass at the level of the cricoid cartilage in the right posterior paramedian region. Also visible are the coarse, stippled calcifications of the tumor, which are characteristic of chondrosarcoma. 


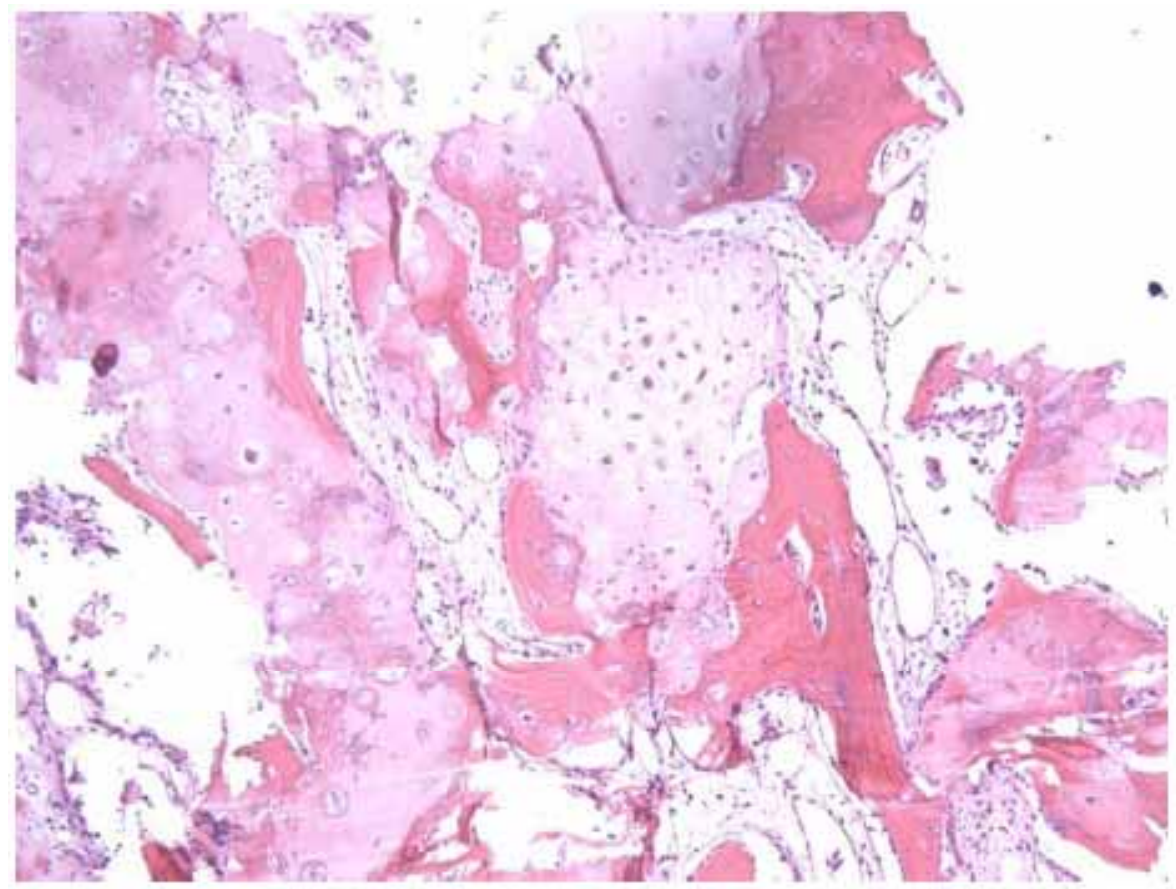

Fig. (3). Low magnification view showing hypercellular areas of cartilage admixed with bone. (H\&E, x100).



Fig. (4). Low-grade (grade 1/3) hyaline type chondrosarcoma comprised of hypercellular hyaline cartilage with mild nuclear atypia and associated areas of enchondral ossification (left). (H\&E, x400).

extent that he was able to resume his pre-operative diet without difficulty. Follow-up CT evaluation 1 year after treatment demonstrated marked reduction in the volume of the tumor mass with residual high-density calcifications (Fig. 6). $\mathrm{He}$ is being followed regularly at six monthly intervals with flexible digital-chip video laryngoscopy and to date, 2.5 years after tumor removal, remains symptom free without evidence of growth.

\section{LITERATURE REVIEW}

Chondrosarcoma of the larynx is a rare condition and there are less than 200 reports in literature since it was first described by Travers in 1816 [3]. The etiology is unknown and the posterior cricoid cartilage lamina is the site of predilection in most cases [2]. It typically affects men over the age of 50 years, the mean age being 60 to 64 years. Hoarse- 


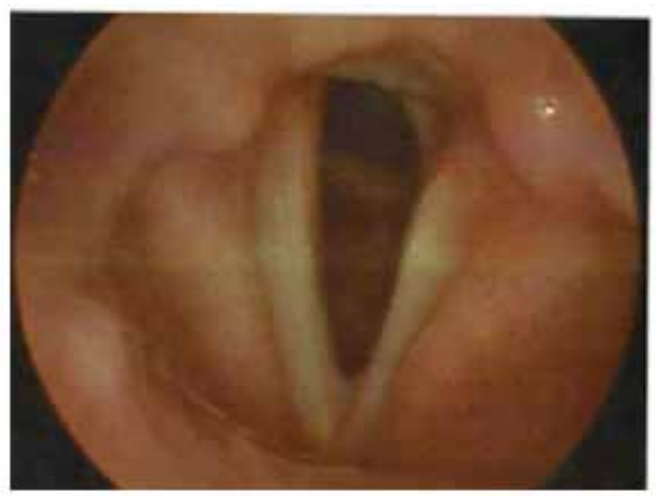

Fig. (5). Follow-up view after excision and completion of radiation therapy. Post-operative view following excision of mass and completion of radiation therapy. The subglottic mass previously seen is markedly diminished in size and the glottic aperture is patent.

ness of the voice is the most common presenting symptom, followed by dyspnea, dysphonia, dysphagia, airway obstruction, and pain. The symptoms are frequently present for a long duration (mean $>2$ yrs) supporting the theory that this is an indolent tumor [4]. This patient did not have any of these signs and he was unaware of any discomfort in the neck prior to having his knee surgery.

The tumor is best diagnosed with a CT scan which characteristically shows a coarse or stippled calcification within the tumor mass in $80 \%$ of the cases [1,2] and this feature was seen in our case (Fig. 2). On mirror examination a smooth but firm, submucosal, lobulated, bluish grey, nonulcerated subglottic mass which may impair normal vocal cord function may be seen.

Laryngeal chondrosarcomas are mostly low-grade neoplasms (grade 1 and 2) histologically and are not clinically aggressive [2]. Less than $5 \%$ are grade 3 type of highly aggressive tumors. Nearly all of them arise from the hyaline cartilage and grading of the tumor is based on pronounced irregularity of the size of the cells and their nuclei, the presence of increased cellularity, nuclear hyperchromasia and binucleated or multi nucleated atypical cells.

Since this arises from the cricoid cartilage, the only complete ring of the trachea, a complete removal can lead to disastrous laryngo-tracheal stenosis in the elderly patient. Without the cricoids cartilage, tracheal collapse can ensue causing respiratory distress. As such, preservation of the cricoids is of the utmost importance in surgical dissection of this area



Fig. (6). Neck computed tomography, following endoscopic removal of tumor and radiation treatment. There is marked decrease in size of the larygngeal mass with residual calcifications, which have remained stable to date. 
during extirpative surgery. To our knowledge, this is mechanistically different from tracheal stenosis in children, but with similar physiologic effects. However, given the multiple potential comorbidities in the elderly, which are mitigating factors, the aberrant physiology is less well-tolerated by elderly patients compared to children. Conservative surgery with all attempts at voice sparing is the primary choice of treatment and can be achieved through endoscopic removal, fissure thyrotomy, partial laryngectomy or rarely total laryngectomy. Recurrence rate is about 35 to $40 \%$ and is related to the grade and incomplete excision [1, 2,5]. Fortunately death from the disease is uncommon and is usually due to local invasion into vital structures $[1,2]$. Distant metastasis, though rare has been reported [6]. Intubation difficulties may be encountered in chondrosarcomas because of the fixed nature of the lesion and the mass effect which narrows the glottic aperture. This is the first report of a difficult intubation secondary to an otherwise asymptomatic laryngeal chondrosarcoma. To our knowledge, there have been no published reports of anesthetic-related deaths related to undiagnosed laryngeal chondrosarcoma. Because these lesions arise directly out of the cricoid ring, the mass lesion is fixed and passing an endotracheal tube on the side of the lesion may lead to deviation of the tube laterally into the pyriform sinus and an esophageal intubation. With vocal fold paralysis, the mobility at the cricoarytenoid joint is preserved, and the glottic aperture can be widened by pushing the vocal fold and arytenoid laterally. Vocal fold immobility secondary to mass lesions pose a more difficult intubation problem because the fixation of the cricoidarytenoid joint will prevent the flexible endotracheal tube from entering the airway. A smaller endotracheal tube and entering the glottic aperture on the opposite of the lesion is necessary to gain access into the trachea.

In conclusion, laryngeal chondrosarcomas can be a cause of unexpected difficult intubation and has not been reported before. As the tumor is sub-glottic it is hard to visualize with direct laryngoscopy and could remain undetected unless it is causing symptoms and the average duration before detection is 1.8 years [7]. We do not know how long our patient had the tumor as it was picked up incidentally during the difficult laryngoscopy and fortunately he is doing well more than 2 years after the diagnosis was made and he has an almost intact laryngeal function.

\section{ACKNOWLEDGEMENTS}

The authors would like to acknowledge the patient, for allowing us to present this case, as well as the Oral and Maxillofacial Surgery Foundation (SMS), Massachusetts General Hospital Department of Oral and Maxillofacial Surgery Education and Research Fund (SMS) and the Massachusetts General Hospital Physicians Organization (SMS, WCF, JS).

\section{REFERENCES}

[1] Thompson LD, Gannon FH. Chondrosarcoma of the larynx: a clinicopathologic study of 111 cases with a review of the literature. Am J Surg Pathol 2002; 26(7): 836-51.

[2] Lewis JE, Olsen KD, Inwards CY. Cartilaginous tumors of the larynx: clinicopathologic review of 47 cases. Ann Otol Rhinol Laryngol 1997; 106(2): 94-100.

[3] Travers F. A case of ossification and bony growth of the cartilages of the larynx, preventing deglutition. Med Chir Trans 1816; 7: 150-3.

[4] Maish M, Vaporciyan AA. Chondrosarcoma arising in the trachea: a case report and review of the literature. J Thorac Cardiovasc Surg 2003; 126(6): 2077-80.

[5] Brandwein M, Moore S, Som P, et al. Laryngeal chondrosarcomas: a clinicopathologic study of 11 cases, including two "dedifferentiated" chondrosarcomas. Laryngoscope 1992; 102(8): 858-67.

[6] Sirota HH, Hurwitz A. Chondrosarcoma of the larynx. AMA Arch Otolaryngol 1952; 56(3): 290-3.

[7] Jones HM. Cartilaginous tumours of the head and neck. J Laryngol Otol 1973; 87(2): 135-51.

\begin{tabular}{lll}
\hline Received: May 28, 2011 & Revised: July 24, 2011 & Accepted: July 26, 2011
\end{tabular}

(C) Sarma et al.; Licensee Bentham Open.

This is an open access article licensed under the terms of the Creative Commons Attribution Non-Commercial License (http://creativecommons.org/licenses/ by-nc/3.0/) which permits unrestricted, non-commercial use, distribution and reproduction in any medium, provided the work is properly cited. 